\title{
Functional Significance of Striatal Responses during Episodic Decisions: Recovery or Goal Attainment?
}

\author{
Sanghoon Han, ${ }^{1}$ Scott A. Huettel, ${ }^{1,2}$ Ana Raposo, ${ }^{4}$ R. Alison Adcock, ${ }^{1,3}$ and Ian G. Dobbins ${ }^{5}$ \\ ${ }^{1}$ Department of Psychology, Yonsei University, Seoul 120-749, Korea, ${ }^{2}$ Department of Psychology and Neuroscience, Duke University, Durham, North \\ Carolina 27708-0086, ${ }^{3}$ Department of Psychiatry and Behavioral Sciences, Duke University, Durham, North Carolina 27710, ${ }^{4}$ Department of Psychology, \\ University of Lisbon, 1649-013 Lisbon, Portugal, and ${ }^{5}$ Department of Psychology, Washington University in St. Louis, St. Louis, Missouri 63130-4899
}

Memory retrieval is typically a goal-directed behavior, and as such, potentially influenced by reinforcement and motivation processes. Although striatal activation is often evident during memory retrieval, its functional significance remains unclear because typical memory paradigms do not control the motivational significance of memory decisions. We used event-related functional magnetic resonance imaging (fMRI) to investigate striatal activation during recognition with and without performance-linked monetary incentives. During initial performance in the absence of incentives, dorsal striatal activation for "Old" memory conclusions nonetheless exceeded that for "New" conclusions regardless of the accuracy of these conclusions. In contrast, subsequent scans paired incentives with either "Old" or "New" conclusions and demonstrated greater activation for whichever judgment was potentially rewarded, both with and without performance feedback. The data demonstrate that striatal activation during recognition judgments does not signal monetary reward receipt, cognitive feedback, or successful episodic retrieval. Instead, it is heavily dependent upon satisfaction of the subjective goals of the observer.

\section{Introduction}

Outside of the laboratory, memory retrieval attempts are usually performed in service of specific goals (Johnson et al., 1993; Shapiro et al., 2006) and successful remembering often leads to reward laden outcomes such as professional advancement. In contrast, human memory experiments usually divorce retrieval and motivation because individual outcomes or net performance do not bear upon the receipt of credit or payment, which is based solely on completing the study, not performing well. During the simplest of memory judgments, episodic recognition, fMRI studies demonstrate that correctly recognized studied items (HITs) yield greater activation than correctly identified novel items [Correct Rejections (CRs)] in a widespread cortical network including left lateral parietal, left rostrolateral prefrontal, medial prefrontal, and posterior midline areas, as well as subcortical regions (Tulving et al., 1994; Rugg et al., 1996; Buckner et al., 1998). The finding is termed the "retrieval success" effect based on the reasonable assumption that the fundamental difference between HITs and CRs, is the presence of retrieved episodic content for the former. However, this characterization tacitly assumes that the motivational significance of "Old" and "New" decisions is similar, which may be unwarranted if subjects perceive the goal of the task as one of maximizing the detection of studied materials. Consistent with this possibility, the retrieval success contrast also implicates the striatum, particularly the caudate nucleus (von

\footnotetext{
Received June 26, 2009; revised Feb. 1, 2010; accepted Feb. 12, 2010.

This work was supported by the National Institute of Mental Health (R01-MH073982 to I.G.D).

Correspondence should be addressed to Dr. Sanghoon Han, Department of Psychology, Yonsei University, Seoul 120-749, Korea. E-mail: sanghoon.han@duke.edu.

DOI:10.1523/JNEUROSCI.3077-09.2010

Copyright $\odot 2010$ the authors $\quad 0270-6474 / 10 / 304767-09 \$ 15.00 / 0$
}

Zerssen et al., 2001; Achim and Lepage, 2005; Iidaka et al., 2006; McDermott et al., 2009; Spaniol et al., 2009), a region more traditionally linked to the motivational significance of actions (Elliott et al., 2000; Knutson et al., 2001; Delgado et al., 2003; De Martino et al., 2009) which receives midbrain dopaminergic input from substantia nigra (Grahn et al., 2009).

The above highlights an ambiguity in the recognition literature suggesting at least two different functional interpretations for the recognition-linked striatal response. First, it may signal successful memory retrieval, consistent with the striatum's known interconnectivity with hippocampal and midline thalamic regions supporting episodic memory (Wittmann et al., 2005; Adcock et al., 2006; Belujon and Grace, 2008; Cohen et al., 2009). We refer to this as the "retrieval-dependent account" to emphasize that the measured striatal response is critically linked to successful retrieval outcomes. The second possibility is that this striatal response does not directly reflect episodic retrieval, but instead reflects the subjects' motivational preference for reaching "Old" as opposed to "New" conclusions within standard testing situations. We refer to this as the "goal-dependent account" and it is an extension of prior research demonstrating that dorsal striatum activation is influenced by the degree to which instrumental behaviors anticipate rewards or positive feedback (Tremblay et al., 1998; Zink et al., 2003; O’Doherty et al., 2004; Tricomi et al., 2004).

To test these hypotheses it is necessary to render "Old" and "New" conclusions both consistent and inconsistent with a priori favored outcomes. We achieved this by crossing monetary incentives with the status of recognition probes (Studied or Novel). If the retrieval-dependent hypothesis is correct, then greater striatal activation for HITs than CRs will occur, regardless of incentive 
A

\begin{tabular}{cc}
\hline RUN Order & \\
\hline STUDY Phase 1 & No Reward or Feedback \\
Recognition TEST 1 & manipulation explained \\
Recognition TEST 2 & \\
STUDY Phase 2 & \\
\hline Instruction & Potential for Reward explained \\
\hline Recognition TEST 3 & "Old" Potentially Rewarded \\
Recognition TEST 4 & "New" Potentially Rewarded \\
\hline
\end{tabular}

The order of reward type in Test 3 and 4 was counterbalanced

B

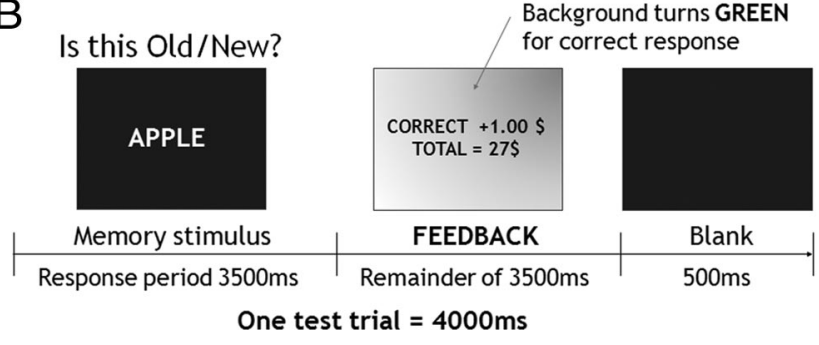

Figure 1. Schematic figures of the experimental paradigm. $\boldsymbol{A}$, Scan run order. $\boldsymbol{B}$, Example trial structure during incentive trial with feedback ("Old" potentially rewarded).

structure. Conversely, if the goal-dependent hypothesis is correct, then differential activation of HITs and CRs will track the incentives, not the memory status of the probes.

\section{Materials and Methods}

Subjects and task materials. Twenty native English-speaking volunteers participated ( 11 females, mean age of 24 years, age range $20-33$ ). One subject was excluded from analysis for failure to complete the experiment. Informed consent was obtained in compliance with the Institutional Review Board of Duke University Medical Center. The participants were paid $\$ 20$ for each hour of participation and an additional maximum of $\$ 40$ based on their recognition performance. For the recognition testing, 500 nouns were drawn randomly from the same word pool (average 7.09 letters, and 2.34 syllables, with a Kucera-Francis corpus frequency of 8.85). From this list, four lists of 100 items (50 OLD, $50 \mathrm{NEW}$ items for each cycle) were constructed for use in two study lists distributed across four scanned recognition tests. One hundred additional items were selected and presented as NEW items during a postscan recognition test. During scanning, stimuli were back-projected onto a viewing mirror. All responses were made with the left hand using a four key optical button box.

Experimental procedure. In total, there were 6 sequential scanning sessions or "scans" for short. During the first four scans, recognition memory was examined. One encoding period preceded recognition scans 1 and 2, and another encoding period preceded recognition scans 3 and 4 . Following these recognition scans, subjects engaged in a non-memory incentive guessing scan, and finally, a postscan surprise behavioral recognition test of the items that were novel during the prior incentive runs. The key parts of the design are illustrated in Figure 1 and described below. Importantly, no mention of the potential for reward was made until just before recognition scan 3 and therefore any activation observed in striatal regions during scans 1 and 2 is not reflective of the expectation of performance linked reward. Data from the final non-mnemonic incentive guessing task is omitted due to a programming error that limited the number of available trials. The encoding periods were not scanned.

During encoding, subjects performed an incidental encoding task, counting the number of syllables for each serially presented word. Each encoding phase had 100 trials supporting two subsequent, scanned test phases. Each word appeared with a task cue "Counting syllables 1/2/3/ $>4$ " underneath. If a subject failed to respond within $2 \mathrm{~s}$ a warning message encouraging quicker responding was presented.

During the initial standard recognition scans (Scans 1 and 2) subjects were presented with old words from the previous encoding list inter- mixed with new words, and judged each serially presented item using four response options (High confident Old-Low confident Old-Low confident New-High confident New). There were a total of 100 test items (50 OLD items $+50 \mathrm{NEW}$ items) in each test scan. Test trials were interspersed with 25 passive fixation trials as determined by an optimal sequencing program (Wager and Nichols, 2003). In addition, there were 20 motor control trials in which subjects were presented a randomly chosen number $(1 \sim 4)$ and instructed to press the corresponding key. The memoranda and response prompt appeared simultaneously for $3.5 \mathrm{~s}$ followed by $0.5 \mathrm{~s}$ blank interval before the next trial [stimulus onset asynchrony (SOA) of $4 \mathrm{~s}$ ]. Again, during scans 1 and 2 there was no mention of any potential reward or delivery of any performance feedback.

Scans 3 and 4 explicitly linked recognition conclusions to potential reward. Studied items were encoded in the interim before scan 3 and the potential for monetary reward in scans 3 and 4 was only divulged after completion of this second study list. This prevented reward contamination of the first two recognition scans and of the study period preceding scans 3 and 4 . Instructions were provided on screen and questions answered via intercom before proceeding with the incentive test conditions. In each scan a different judgment was associated with potential reward and punishment. For the HIT-Incentive scan, subjects were informed that correct "Old" responses (HIT) would potentially receive 1 dollar whereas incorrect "Old" responses would potentially lose a dollar [False Alarms (FA)]. They were further instructed that "New" responses could neither earn nor lose money [Correct Rejections (CR) or Misses (MS)]. This corresponds to a neutral payout under maximum expected value in signal detection decision models (Macmillan and Creelman, 1991) and hence should not appreciably move the decision criterion. For the CRIncentive scan these payoff contingencies were reversed with CRs potentially earning a dollar and MSs potentially losing a dollar; "Old" reports were neither potentially rewarded nor punished. The order of these scan types was counterbalanced across subjects.

The second key manipulation during these two scans was whether feedback followed responding for responses linked to the incentive condition. On $\sim 50 \%$ of the incentive concordant (e.g., "Old" during HIT-Incentive scan) trials the subjects received immediate feedback indicating response accuracy and a running total of reward accrued, whereas the remainder did not include feedback and reward accrual information. The purpose of the no-feedback condition was to see whether the recognition judgments continued to evoke activation in striatal regions even in the absence of external feedback delivery. Subjects were instructed that the final payout was not linked to whether or not a given incentive concordant response elicited feedback, thus failing to receive feedback for an incentive-concordant response did not mean that it was not rewarded (or punished). The delivery of feedback was determined based on the subjects' report using a random number generator to ensure that approximately half of the incentive concordant responses received feedback. To ensure that participants did not forget which response was incentivized a reminder prompt remained upon screen throughout the test (e.g., "OLD ITEMS EARN MONEY"). Participants were instructed that one of the two scans would be chosen by coin flipping to determine the actual payout at the end of the experiment and that they would earn either their winnings or maximum 40 dollars whichever was lower.

The trial structure of incentive scans was similar to that of the initial standard recognition scans, except that the reward feedback was displayed immediately after responding until the blank ( $0.5 \mathrm{~s})$ ITI period was initiated (Fig. $1 B$ ). Outcome and reward information were indicated using text and screen coloring during feedback trials. When subjects responded during no-feedback trials a series of X's appeared on the background where the reward information was typically displayed, and the background turned gray instead of red (incorrect response) or green (correct response). As in scans 1 and 2, the SOA remained at 4 s. Passive fixations and active control trials were presented and sequenced as in scans 1 and 2 .

Following all scanning and before payment, subjects were given a final behavioral recognition test for the new items encoded during the two incentivized recognition scans. The key question addressed was whether the reward conditions affected the quality of encoding of the new items 
during testing. There were 100 target items consisting of all of the new items from the two scans. The test used the same recognition test format as the one during scanning except there was no reward. Finally, the BIS/BAS (behavioral inhibition system/behavioral activation system) personality inventory was given before completing the experiment (Carver and White, 1994).

fMRI acquisition. Scanning was performed on a 3T General Electric scanner using a standard head coil. Functional data were acquired by using a gradient echo echo-planar pulse sequence $(\mathrm{TR}=2000, \mathrm{TE}=31$ ms, 34 axial slices parallel to the AC-PC plane with near-isotropic voxels of $3.75 * 3.75 * 3.8 \mathrm{~mm}$, no gap, interleaved collection). Before functional data collection, four dummy volumes were discarded to allow for equilibration effects. Participants' head motion was minimized by using foam padding. High-resolution T1-weighted anatomical images [three-dimensional spoiled gradient recalled acquisition (SPGR)] were acquired for visualization.

fMRI data preprocessing and analyses. Data were processed using SPM2 (Wellcome Department of Cognitive Neurology, London, UK). Slice acquisition timing was corrected by resampling all slices in time relative to the middle slice collected, followed by rigid body motion correction across all scans. Functional data were spatially normalized to a canonical echo-planar imaging (EPI) template using a 12-parameter affine and nonlinear cosine transformation, with volumes then resampled into 2 $\mathrm{mm}$ cubes and spatially smoothed with an $8 \mathrm{~mm}$ fullwidth at halfmaximum isotropic Gaussian kernel. Each scanning session was rescaled such that the mean global signal was 100 across the volumes. For the analyses, volumes were treated as a temporally correlated time series and modeled by convolving a canonical hemodynamic response function (HRF) and its temporal derivative with a delta function marking each trial onset. The resulting functions were used as covariates in a general linear model, along with a basis set of cosine functions that were used to high-pass filter the data and a covariate representing session effects. The least squares parameter estimates of the best-fitting synthetic HRF for each condition of interest (averaged across scans) were used in pairwise contrasts and stored as a separate image for each subject. These difference images were then tested against the null hypothesis of no difference between contrast conditions using either one-tailed $t$ tests or nondirectional $F$ tests. The data were statistically analyzed treating subjects as a random effect. Unless stated otherwise, effects were considered significant if they exceeded an uncorrected threshold of $p<0.001$ and consisted of five or more contiguous voxels.

Functional regions of interest (ROIs) were extracted using the MarsBar Toolbox (Brett et al., 2002) using coordinates obtained from the SPM contrast maps. Average parameter estimates were obtained for the significant voxels within an $8 \mathrm{~mm}$ radius of each of the SPM-identified maxima and further analyzed using off-line statistical software. Responses from the standard recognition/No-Incentive conditions were averaged across scans 1 and 2.

Competitive prediction analysis. A form of functional connectivity using multiple regression was used to directly pit two ROIs, caudate and rostrolateral prefrontal cortex (PFC), against one another in terms of their ability to predict task evoked responses in target regions, across participants. The selection of rostrolateral PFC was based on its reliable link with episodic retrieval in prior functional imaging literature (Lepage et al., 2000; McDermott et al., 2000; Rugg and Wilding, 2000). Furthermore, the region also has been linked with top-down cognitive control such as relational reasoning or planning processes that are prospective (Christoff and Gabrieli, 2000; Dobbins and Han, 2006; Reynolds et al., 2006; Wendelken et al., 2008). Thus, given its prior role in top-down cognitive control, episodic retrieval and higher order reasoning, we predicted that the rostrolateral PFC region might associate with a different network than striatal regions implicated by the incentive manipulation and previously shown to be sensitive to the anticipation of reward (Knutson et al., 2001).

To test this we used a functional connectivity method that we term competitive prediction analysis to examine the interrelationship between the different ROIs of the global F-map (Fig. 2) across participants. The only difference between standard functional connectivity methods and the current method is that the former typically use only a single seed

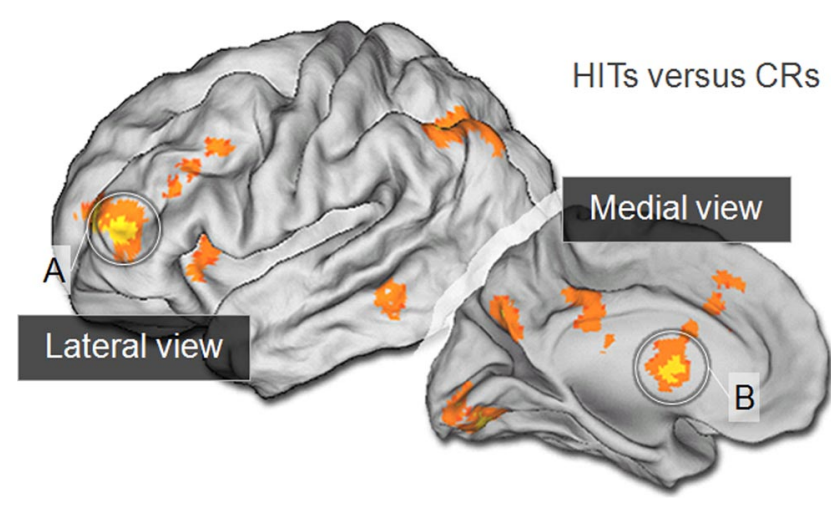

Figure 2. F-analysis whole brain activation during the retrieval success effect (HITs versus (Rs). Left-hemisphere lateral and medial activations are mapped onto the PALS-B12 atlas (Van Essen, 2005). Circled are the two critical regions of interest, left rostrolateral $\operatorname{PFC}(\boldsymbol{A})$ and caudate nucleus $(\boldsymbol{B})$.

region and examine its simple correlation with other regions (Rissman et al., 2004). In contrast, this approach uses multiple regression with striatal and rostrolateral PFC seed regions entered simultaneously, thereby capturing the unique contribution of the two ROIs (if any) to the prediction of other target regions in the map. Thus the analysis addresses whether a given subject's tendency to differentially activate caudate and rostrolateral PFC ROIs, in isolation or conjunction, predicts activation in tertiary regions. Furthermore, it measures the degree to which each predictor region carries unique variance with respect to the activation response in tertiary regions. If caudate and rostrolateral PFC are functionally separable then this analysis will yield solutions where one or the other region uniquely predicts activity in a third region, or cases where they both significantly contribute to predicting a third region's response. In contrast, if they are part of a single highly integrated network they will carry largely redundant predictive information and hence generally fail to predict activation in tertiary regions. This approach examined the degree to which the HITs $>$ CRs activation, as estimated by the $\beta$ difference for each subject, in rostrolateral PFC and caudate were predictive of the remainder of ROIs defined from the retrieval success map. The analysis was restricted to the initial No-Incentive recognition runs where subjectbased differences in strategies were likely to be most prominent.

\section{Results}

\section{Behavioral results}

One-way within-subjects ANOVA demonstrated that neither accuracy $\left(d^{\prime}\right)$ nor response bias $(c)$ differed across the three incentive conditions $\left(F_{(2,36)}=0.96, p=0.38 ; F_{(2,36)}=1.26, p=0.28\right)$. However, decision confidence was affected (supplemental Table 1, available at www.jneurosci.org as supplemental material). More specifically, when a memory judgment was in conflict with the potential to earn reward, it tended to be rendered less confidently than when it was congruent with the external incentive, or there was no incentive present. A Response Type (HITs, CRs) by Incentive Condition (No-Incentive, HIT-Incentive, CR-Incentive) ANOVA on average confidence levels yielded main effects of Response Type $\left(F_{(1,18)}=6.43, p<0.05\right)$ and Incentive Condition $\left(F_{(2,36)}=3.29\right.$, $p<0.05)$, and an interaction between these factors $\left(F_{(2,36)}=\right.$ $18.08, p<0.001$ ) (supplemental Table 1, available at www.jneurosci. org as supplemental material). Pairwise contrasts (Fisher's LSD) demonstrated that HITs were more confident than CRs under the No-Incentive condition $(p<0.01)$. This difference remained under the HIT-Incentive condition $(p<0.001)$, and it was reversed under the CR-Incentive condition ( $p=0.051)$ (supplemental Table 1, available at www.jneurosci.org as supplemental material). 
The pattern of reaction times for HITs and CRs was also influenced by the incentive conditions, suggesting quicker responding for incentive congruent decisions. A Response Type (HITs, CRs) by Incentive Condition (No-Incentive, HITIncentive, CR-Incentive) ANOVA on mean reaction times yielded no main effect of Response Type $(F<1)$, a main effect of Incentive Condition $\left(F_{(2,36)}=25.09, p<0.001\right)$ and an interaction between these factors $\left(F_{(2,36)}=9.43, p<0.001\right)$ (supplemental Table 1, available at www.jneurosci.org as supplemental material). Pairwise contrasts (Fisher's LSD) demonstrated that HITs were rendered more quickly than CRs under the NoIncentive condition $(p<0.01)$. The numerical advantage for HITs remained in the HIT-Incentive condition although it was unreliable $(p=0.31)$. Under the CR-Incentive condition CRs were now rendered more quickly than HITs $(p<0.01)$.

Overall, the behavioral data demonstrate that while the incentives did not alter overall accuracy or bias, they did alter the confidence and speed with which decisions were rendered. Under the incentive conditions, subjects tended to respond more quickly and confidently when decisions were congruent with the incentives in place. Under the No-Incentive condition, HITs were rendered more confidently and quickly than CRs. Hit rates during the final postscan recognition test are also listed in supplemental Table 1, available at www.jneurosci.org as supplemental material. There was no difference in subsequent hit rates for previously novel items drawn from HIT-Incentive scan and from CR-Incentive scan suggesting that the different incentives did not affect the quality of encoding of novel materials.

\section{Functional MRI results}

Whole brain analyses

The first analysis was conducted to confirm that striatal regions were implicated in the standard retrieval success contrast (HITs $>$ CRs) during the No-Incentive conditions. Greater activation for HITs relative to CRs was observed in bilateral caudate as well as other regions typically associated with this contrast (Table 1, condition A). Next, to provide unbiased regions of interest (ROIs) for further investigation, the HITs versus CRs contrast was subjected to a nondirectional $F$ test collapsed across the factors of Incentive Condition (No-Incentive, HIT-Incentive, CR-Incentive) and Feedback Presence (Present, Absent). The analysis identified regions including left rostrolateral PFC, superior medial PFC, posterior cingulate, precuneus, left lateral parietal regions, and bilateral lingual gyrus (Fig. 2, Table 1, condition B). Critically, the analysis also demonstrated prominent bilateral activations of medial caudate. The ROI-based analyses below examine these responses more fully focusing primarily on bilateral caudate (Fig. 3) and contrasting its response with rostrolateral PFC.

\section{ROI analyses: recognition under incentives}

Examination of the mean $\beta$ s drawn from the bilateral caudate ROIs (head of caudate; Fig. 3) suggested three important effects (see supplemental Data for analogous patterns in each left and right caudate ROI and from nearby regions in the body of the caudate). First, the HITs $>$ CRs pattern obtained during standard recognition (No-Incentive) was amplified during the HITIncentive run, even when restricting consideration to trials without feedback (Fig. 3A, B, light gray and white bars). This was confirmed via a significant two-way interaction between Incentive Condition (No-Incentive, HIT-Incentive) and Response Type (HITs, CRs) $\left(F_{(1,18)}=15.40\right.$, MSe $\left.=1.43, p<0.001\right)$. Second, the caudate response to HITs and CRs during incentive runs tracked the external incentives and not the item's
Table 1. Regions demonstrating greater activation during HITs versus CRs during No-Incentive condition (condition A) and during $F$ test with all conditions (condition B)

\begin{tabular}{|c|c|c|c|c|c|c|}
\hline Regions & Lat. & BA & $x$ & $y$ & $z$ & $z$-score \\
\hline \multicolumn{7}{|l|}{ HITs $>$ CRs No-Incentive condition } \\
\hline Medial frontal gyrus & $\mathrm{L}$ & $6 / 8 / 32$ & -6 & 26 & 46 & 3.25 \\
\hline Rostrolateral frontal & $\mathrm{L}$ & 10 & -42 & 50 & 4 & 3.55 \\
\hline \multirow[t]{2}{*}{ Cingulate gyrus } & $\mathrm{L}$ & 31 & -4 & -44 & 40 & 3.57 \\
\hline & R & 31 & 10 & -44 & 40 & 3.55 \\
\hline Lingual gyrus & $\mathrm{L}$ & 18 & -18 & -78 & -6 & 3.88 \\
\hline Cuneus & $\mathrm{L}$ & 7 & -6 & -72 & 32 & 3.40 \\
\hline Superior/inferior parietal lobule & $\mathrm{L}$ & $7 / 40$ & -44 & -60 & 50 & 4.08 \\
\hline Precuneus & $\mathrm{L}$ & 19 & -34 & -80 & 46 & 4.90 \\
\hline \multirow[t]{5}{*}{ Caudate } & $\mathrm{L}$ & & -8 & -2 & 22 & 3.48 \\
\hline & & & -6 & 4 & 14 & 3.30 \\
\hline & & & -6 & 8 & 4 & 3.25 \\
\hline & R & & 8 & -2 & 20 & 3.62 \\
\hline & & & 8 & 12 & 22 & 3.51 \\
\hline Middle/inferior temporal gyrus & $\mathrm{L}$ & $20 / 37$ & -56 & -38 & -20 & 3.23 \\
\hline \multicolumn{7}{|l|}{ HITs $>$ CRs F-map all conditions } \\
\hline \multirow{2}{*}{ Lingual gyrus } & $\mathrm{L}$ & $18 / 19$ & -20 & -82 & -14 & 5.07 \\
\hline & $\mathrm{R}$ & $18 / 19$ & 16 & -70 & -6 & 4.85 \\
\hline \multirow[t]{6}{*}{ Caudate* } & $\mathrm{L}$ & & -8 & 4 & 6 & 4.92 \\
\hline & & & -10 & 6 & 18 & 4.10 \\
\hline & $\mathrm{R}$ & & 8 & 4 & 8 & 4.31 \\
\hline & & & 8 & 12 & 22 & 4.23 \\
\hline & & & 12 & 8 & 16 & 4.01 \\
\hline & & & 14 & -6 & 22 & 3.33 \\
\hline \multirow[t]{2}{*}{ Thalamus } & $\mathrm{L}$ & & -8 & -26 & 20 & 3.66 \\
\hline & $\mathrm{R}$ & & 6 & -6 & 2 & 3.77 \\
\hline Colliculus/brainstem & & & -2 & -28 & 2 & 3.79 \\
\hline Insula & $\mathrm{L}$ & $13 / 47$ & -34 & 18 & 2 & 3.73 \\
\hline Superior/medial frontal gyrus & $\mathrm{L}$ & $6 / 8 / 9 / 32$ & -6 & 26 & 44 & 3.58 \\
\hline Rostrolateral frontal $^{a}$ & $\mathrm{~L}$ & 10 & -42 & 44 & 4 & 4.44 \\
\hline Middle frontal gyrus & $\mathrm{L}$ & 46 & -44 & 32 & 20 & 3.63 \\
\hline \multirow[t]{2}{*}{ Inferior frontal gyrus } & $\mathrm{L}$ & 47 & -28 & 22 & -6 & 3.63 \\
\hline & $\mathrm{R}$ & $11 / 47$ & 32 & 26 & -8 & 4.02 \\
\hline Superior parietal lobule & $\mathrm{L}$ & 7 & -30 & -68 & 48 & 4.09 \\
\hline Inferior parietal lobule & $\mathrm{L}$ & $39 / 40$ & -50 & -58 & 50 & 4.36 \\
\hline \multirow[t]{2}{*}{ Precuneus } & L & $7 / 19$ & -6 & -72 & 36 & 4.15 \\
\hline & $\mathrm{R}$ & 7 & 14 & -62 & 20 & 4.08 \\
\hline Angular gyrus & $\mathrm{L}$ & 39 & -44 & -76 & 40 & 3.41 \\
\hline Superior temporal gyrus & L & 41 & 52 & -22 & 12 & 3.38 \\
\hline Middle temporal gyrus & $\mathrm{L}$ & $21 / 20$ & -64 & -42 & -10 & 3.86 \\
\hline Posterior cingulate & $\mathrm{L}$ & 23 & -4 & -34 & 30 & 3.65 \\
\hline Anterior cingulate & $\mathrm{L}$ & 33 & -6 & 12 & 24 & 4.10 \\
\hline
\end{tabular}

BA, approximate Brodmann's locations. $x, y$, and $z$ correspond to the MNI coordinates of the maximum voxel. Average $z$-score from SPM map within an $8 \mathrm{~mm}$ radius of maxima.

${ }^{a}$ Regions for ROI analyses.

memory status, even in the absence of feedback (Fig. $3 B, C$, light gray and white bars). This was confirmed by a two-way interaction across Incentive Condition (HIT-Incentive, CRIncentive) and Response Type (HITs, CRs) that revealed a full crossover $\left(F_{(1,18)}=41.04, \mathrm{MSe}=1.85, p<0.001\right)$, with CRs now eliciting relatively greater activation than HITs during the CRIncentive run $\left(t_{(1,18)}=3.50, p<0.005\right)$. Finally, the caudate was sensitive to the presence of feedback, which amplified the response of incentive concordant decisions. Thus HITs followed by feedback elicited greater activation than HITs without feedback during the HIT-Incentive run $\left(t_{(1,18)}=4.70, p<0.001\right.$; Fig. $3 B$, dark gray and light gray bars) and CRs accompanied by feedback yielded greater activation than those without feedback during the CR-Incentive run $\left(t_{(1,18)}=2.78, p<0.05\right.$; Fig. $3 C$, black and white bars). Thus the caudate response was governed primarily by the match between incentives and judgments, and not the 


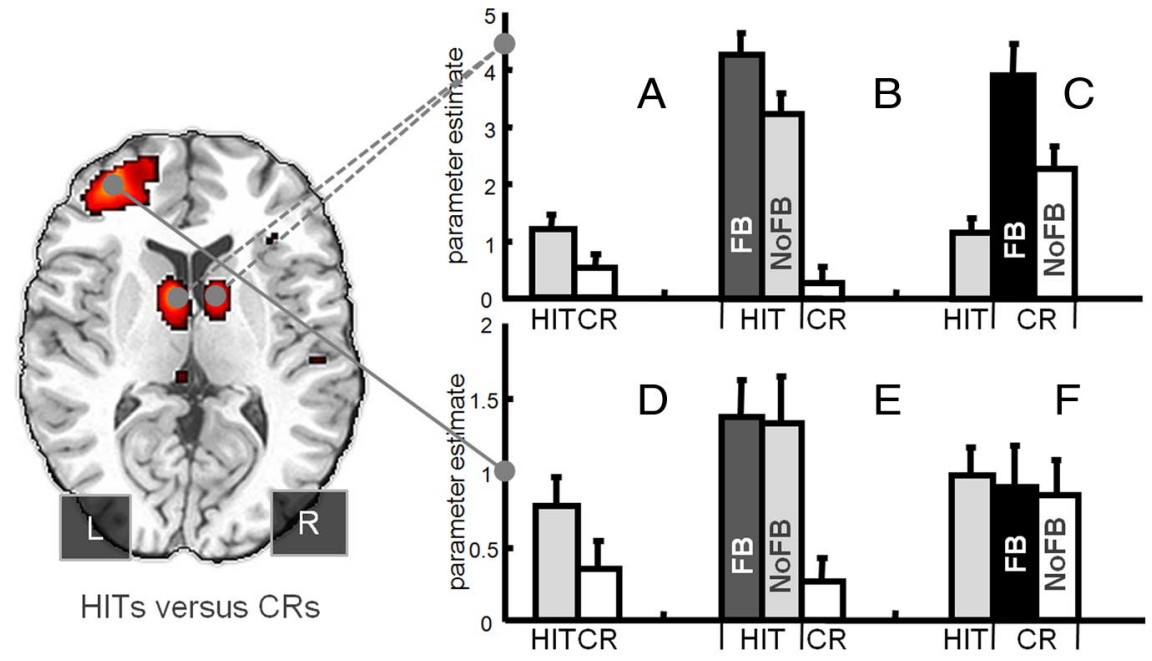

No-Incentive HIT-Incentive CR-Incentive

Figure 3. The mean and SEs of the parameter estimates extracted from the bilateral caudate (top, $\boldsymbol{A}-\boldsymbol{C}$ ) and left rostrolateral PFC (bottom, D-F) ROls. FB, Feedback; NF, no-feedback.
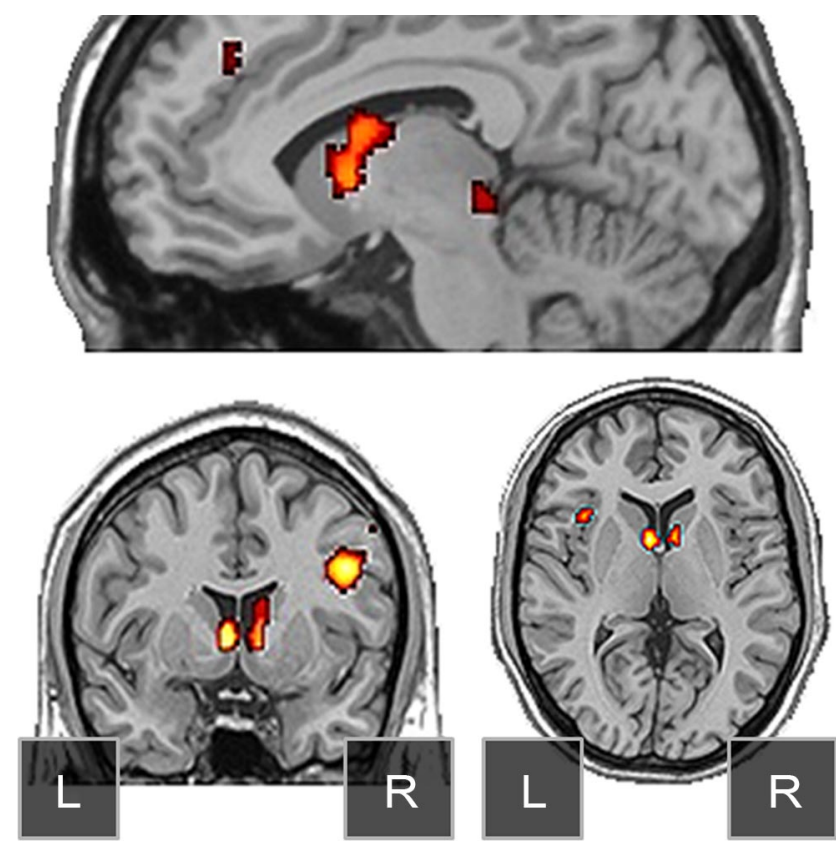

Figure 4. Conjunction images demonstrating regions of pure incentive effects include bilateral caudate nucleus, colliculus, right posterior dorsolateral PFC, and left anterior insula.

memory status of the probe suggesting the region tracks the motivational significance of the decisions. Although having the NoIncentive runs before Incentive runs presents a minor order confound, it does not affect the critical findings. More specifically, the latter two incentive runs in the current study are fully counterbalanced and yet the striatal ROI analyses demonstrated a clear crossover interaction across Response Type and Incentive Condition that practice or order cannot explain.

The left rostrolateral PFC ROI displayed a qualitatively different pattern from the caudate (Fig. 3D-F). Although this region did demonstrate an amplification of the HITs $>$ CRs activation difference when transiting from the No-Incentive to HITIncentive conditions $\left(F_{(1,18)}=4.51, \mathrm{MSe}=0.44, p<0.05\right)$, it did not demonstrate the crossover pattern between the Hit-Incentive and CR-Incentive conditions, and it was insensitive to the presence

versus absence of feedback. The failure to achieve an incentive-based crossover is shown in Figure $3 F$ where activation to the incentivized CRs was numerically lower, not higher than HITs ( $p>0.45)$. The insensitivity to feedback was demonstrated both during the Hit-Incentive run (Fig. $3 E$; $t<$ $0.16, p>0.87$ ) and CR-Incentive run (Fig. $3 F ; t<0.42, p>0.67)$. These qualitative differences survived direct comparison across the two separate regions such that feedback modulated HITs in the caudate but not left rostrolateral PFC during HITIncentive conditions (Fig. $3 B$ vs $E ; F_{(1,18)}=$ 6.58, $\mathrm{MSe}=0.90, p<0.05)$. Likewise, feedback modulated CRs in caudate but not left rostrolateral PFC during CR-Incentive conditions (Fig. $3 C$ vs $F ; F_{(1,18)}=7.54$, MSe $=1.45, p<0.05)$. Furthermore, CRs yielded greater signal than HITs during CR-Incentive conditions in caudate but not left rostrolateral PFC as confirmed by a significant Response Type (HIT, CR) by Region (Caudate, rostrolateral PFC) interaction (Fig. $3 C$ vs $F$ ) in the absence of feedback $\left(F_{(1,18)}=14.30, \mathrm{MSe}=0.56, p<0.01\right)$ or with feedback $\left(F_{(1,18)}=41.99, \mathrm{MSe}=0.90, p<0.001\right)$.

The ROI analyses above indicate that the caudate activation does not signal successful memory retrieval and instead tracks the concordance between judgments and external incentives during the latter incentive scans. To identify other regions potentially demonstrating this full crossover pattern a cross-over conjunction map was constructed that isolated activation regions of HITs (No-Feedback trials) $>$ CRs under the Hit-Incentive condition, and regions of CRs (No-Feedback trials) $>$ HITs under the CRIncentive condition, both at the standard whole-brain-corrected threshold $(0.001 * 0.001,5$ voxels $)$. This conjunction map supported the ROI-based results, clearly implicating bilateral caudate nucleus regions. This map also implicated right posterior lateral PFC, superior colliculus, and left anterior insula (Fig. 4), demonstrating that the responses are not indicative of episodic retrieval, but instead reflect the rendering of judgments consistent with the incentives.

\section{Further interregional dissociations}

The above results demonstrate that caudate and rostrolateral PFC responded differently to the experimental manipulations. This functional dissociation was further tested using an analysis we term competitive prediction analysis. This analysis focused on the initial recognition scans and directly pitted the two regions against one another in terms of their ability to predict the differential HITs $>$ CRs response in other areas, across participants (see Materials and Methods for more details). Table 2 and Figure 5 illustrate the findings, namely, situations in which only the caudate was significantly predictive of a target region (Fig. 5, blue), situations in which only rostrolateral PFC was significantly predictive of a target region (Fig. 5, red), and situations in which both substantially contributed to prediction (Fig. 5, green). Left caudate uniquely predicted activation in the remainder of caudate, colliculus, bilateral anterior insulae, and precuneus. Left rostrolateral $\mathrm{PFC}$ uniquely predicted activation in the remainder of left anterior PFC, left posterior dorsolateral PFC, and the anterior portions of the left inferior parietal lobule (IPL). Finally, 
Table 2. Illustrative findings of competitive prediction analyses (full table available on request)

\begin{tabular}{|c|c|c|c|c|c|}
\hline Prediction pattern & Target & Caudate $\beta$ & $\operatorname{RLPFC} \beta$ & SE $\beta$ & $R^{2}$ \\
\hline \multirow[t]{2}{*}{ A, RLPFC Adv. } & L. IFS $(-422216)$ & $0.27(0.118)$ & $0.65(0.001)$ & 0.16 & 0.61 \\
\hline & L. Ant. Par. $(-50-5445)$ & $0.10(0.640)$ & $0.61(0.008)$ & 0.20 & 0.41 \\
\hline \multirow[t]{2}{*}{ B, Caudate Adv. } & L. Insula (-34 181$)$ & $0.56(0.010)$ & $0.24(0.223)$ & 0.19 & 0.45 \\
\hline & R. Precun. $(16-4536)$ & $0.72(0.001)$ & $0.12(0.500)$ & 0.17 & 0.59 \\
\hline \multirow[t]{2}{*}{ C, Joint Pred. } & Med. PFC (-6 2736$)$ & $0.40(0.016)$ & $0.61(0.001)$ & 0.15 & 0.68 \\
\hline & L. Post. Par. $(-40-5647)$ & $0.40(0.017)$ & $0.61(0.001)$ & 0.15 & 0.67 \\
\hline
\end{tabular}

L., Left; R., right; Med., medial; Post., posterior; Par., parietal; IFS, inferior frontal sulcus; RLPFC, rostrolateral PFC; Adv., advantage; Pred,. prediction.

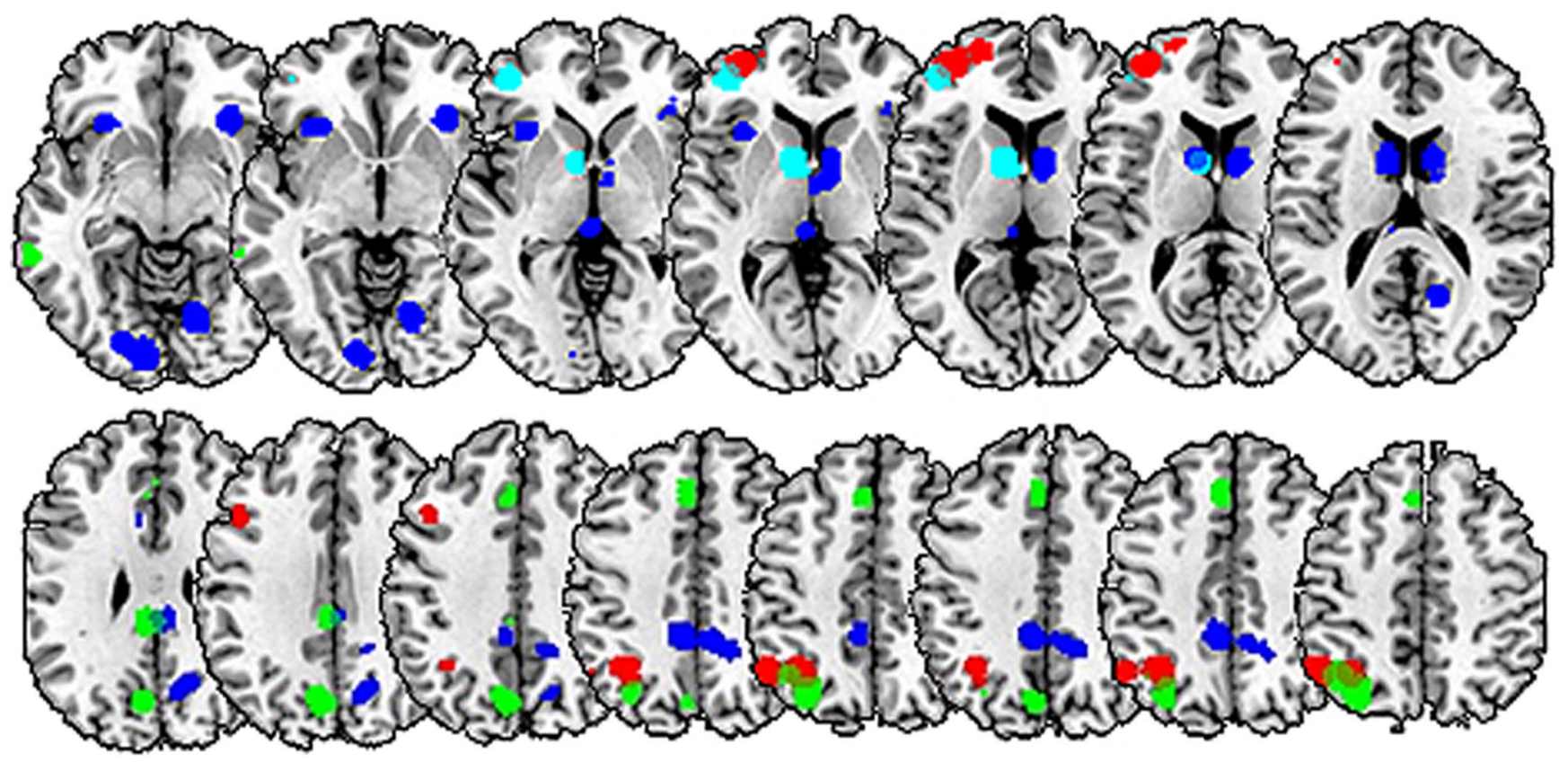

Figure 5. Competitive prediction analysis. Left rostrolateral PFC and caudate seed regions (cyan) were contrasted in their ability to predict activation in tertiary regions of interest across subjects via multiple regression. Red regions illustrate ROIs where the rostrolateral PFC was uniquely predictive, whereas blue regions illustrate regions where the caudate was uniquely predictive. Activation in green regions was jointly predicted by the two seeds. The threshold for analysis was 0.05 .

the regions jointly predicted activation in SMA/anterior cingulate, left posterior IPL and posterior midline.

These data confirm that the two seed regions are part of dissociable but partially overlapping networks and bolster the condition dissociations noted above. Furthermore, the pattern of dissociation is consistent with prior literature. For example, the anterior insula has been linked with reward and risk processing (Preuschoff et al., 2008) and is often considered part of a larger reward processing circuit in which the caudate also plays a central role (Clark et al., 2009). This is consistent with the unique caudate prediction in the current analysis. In contrast, rostrolateral PFC is typically associated with higher order reasoning and known to principally project to other supramodal PFC areas (Dumontheil et al., 2008) and this is consistent with the rostrolateral PFC advantage in predicting activation in left posterior dorsolateral PFC, another area linked to working memory and executive control (Koechlin and Summerfield, 2007). In contrast, the medial SMA/anterior cingulate receives converging input form cortical and subcortical structures (Nakano et al., 2000) and this is consistent with the joint association shown in Table 2.

\section{Personality differences in reward responsiveness}

The above findings indicate that dorsal striatal activations consistent with those historically observed during standard recognition were heavily influenced by incentives and are part of a larger network responsive to reward and motivation. To further inves- tigate this, we examined the potential link between striatal response and the reward responsiveness (RR) subscale of the BIS/ BAS personality scale (Carver and White, 1994), an instrument previously shown to correlate with the striatal BOLD response to incentives during games of chance (Locke and Braver, 2008) and with ventral striatal gray matter density (Schweinhardt et al., 2009). The RR subscale measures self-reported valuation of reward receipt (e.g., "When I get something I want, I feel excited.").

Two approaches were taken, both of which focused on the No-Incentive runs. Analyses were focused on these runs because the variance in the expression of individual, personality-linked responses is likely to be more pronounced in unstructured or ambiguous contexts. In other words, because the reward structure is externally controlled and highly salient in the latter runs, this would likely minimize individual differences linked to personality. First we considered whether activations during recognition in the predefined bilateral caudate or left rostrolateral PFC ROIs were correlated with this independent personality measure. The correlation between the HITs $>$ CRs signal difference and BAS-RR scores approached significance in left caudate ROI ( $r=$ $0.41, p=0.081)$ and reached significance in right caudate $(r=$ $0.54, p<0.05$ ) (Fig. 6A). In contrast, the correlation was negative and nonsignificant in the left rostrolateral PFC ROI $(r=-0.33)$. For both caudate ROIs, these correlations were significantly greater than that in the rostrolateral PFC corre- 
A
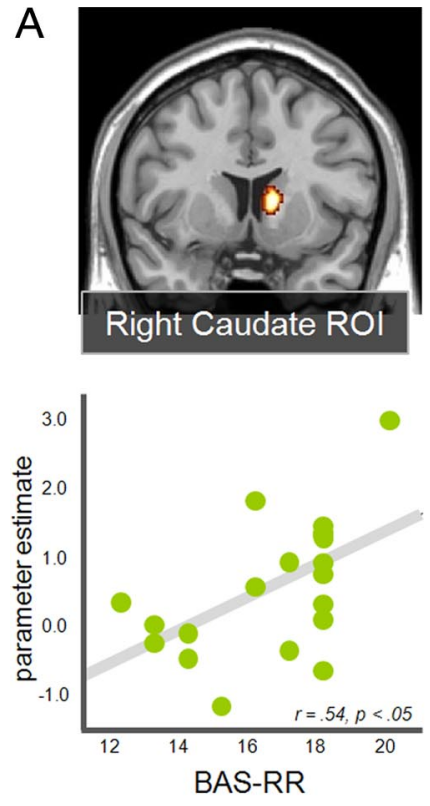

B
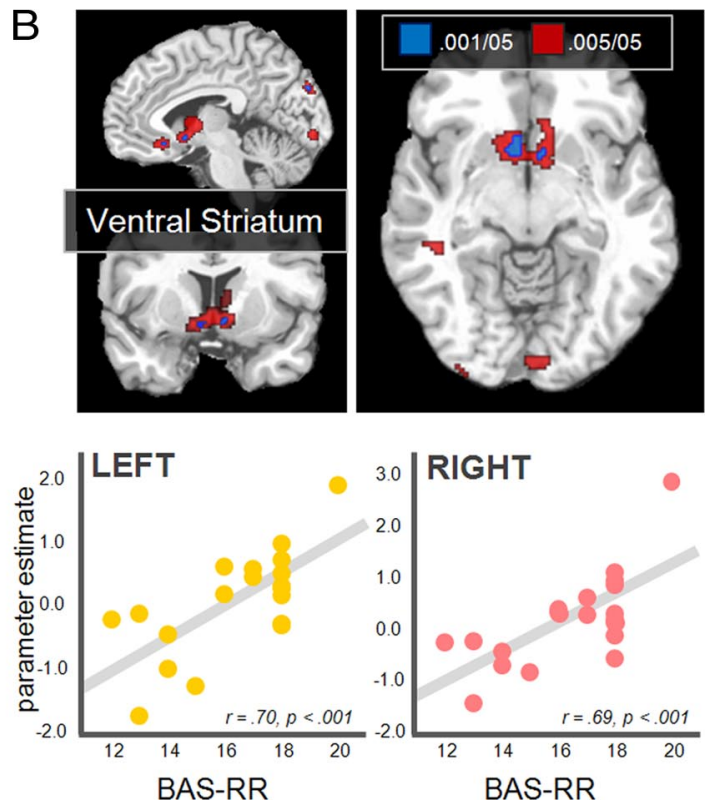

Figure 6. Regions showing individual differences in reward responsiveness. $A$, Right caudate nucleus ROls showing significant positive correlations between HITs $>$ CRs activation and BAS Reward Responsiveness (BAS-RR). $\boldsymbol{B}$, A whole brain simple regression analysis between HITs $>$ (Rs responses and BAS-RR revealed bilateral ventral striatum (voxels significant at $p<0.001$ in blue). Voxels significant at $p<0.005$ are also shown in red to illustrate the extent of activation in caudate regions. L, Left, R, right.

lations ( $p$ values $<0.05$ ). In the second approach the BAS-RR score was entered as a covariate for each subject in a whole brain simple regression analysis searching for regions where the HITs $>$ CRs signal difference correlated with the BAS-RR score. This analysis detected a significant relationship in bilateral ventral striatum and basal forebrain regions at the standard threshold (Fig. $6 B$ ). When the threshold was relaxed to 0.005 the extent of activation increased in these areas, extending dorsally into caudate regions. We note that these same personality-linked findings were not present in the subsequent external incentive runs, suggesting that they were overshadowed when the experimental design renders incentives external and/or unambiguous.

\section{Discussion}

The greater striatal fMRI activation during HITs compared to CRs in standard recognition designs has, in isolation, been difficult to explain, given that recognition tasks are usually devoid of feedback and do not differentially reward these two judgments. The current findings a) clarify the presence of striatal responses during standard recognition tasks, b) differentiate striatal responses from more traditional recognition-linked cortical responses, and c) illustrate the influence of incentives on recognition confidence and speed. Critically, in the current data as in prior recognition literature, there is an increased response in striatal regions during HITs compared to CRs during standard recognition despite the fact that the two response types are not differentially rewarded and there is no expectation of differential reward on the part of the subjects.

Under the goal-dependent account it is assumed that observers subjectively value "Old" more than "New" judgments during discrimination problems described as tests of their recognition memory abilities. The current data further support the goal-dependent account by showing that when incentives were manipulated to differentially associate either "Old" or "New" judgments with potential reward, differential activity in several regions (Figs. 2, 4) tracked the incentives independent of the memory status of the probes.
Subjects demonstrated a greater activation for whichever judgment had the potential to earn reward and this effect was prominent even on trials without performance feedback, although such feedback significantly amplified the response (Fig. $3)$. Together, these results indicate that striatal activation during recognition is largely, if not wholly, dependent upon the observer reaching conclusions consistent with their favored outcomes.

Although goal constructs are not typically considered important in human functional imaging memory research, they have been regarded important in the behavioral memory literature (Johnson et al., 1993) and it is becoming increasingly clear that understanding the link between subjective motivation and memory decisions can be essential to understanding either domain. For example, Tricomi and Fiez (2008) scanned subjects during a paired-associate learning task in which the associations were learned across three trials. Significant caudate activation was observed on the second and third trials, where subjects could use their memory for the prior outcomes to inform the judgment, but not on the first trial even when a correct response was indicated by feedback (Tricomi and Fiez, 2008). The lack of activation during the first round of trials, thus, may reflect the absence of mnemonic information that signals goal achievement. Somewhat similarly, recent work in the rat has demonstrated increased ventral striatal firing during maze learning at choice points that well precede the delivery of reward (van der Meer and Redish, 2009). Thus early in the learning of the correct maze route, striatal activation increased at points where the animals were presumably making memory-based decisions even though these decisions necessarily preceded the actual delivery of reward. These findings along with the current data suggest that the striatal responses can reflect memory-based decisions, in so far as those decisions anticipate the delivery of reward or the satisfaction of a priori goals. Critically, the current design demonstrates that during human retrieval decisions there need not be an expectation of extrinsic reward and that even when an extrinsic reward potential is present (Hit-Incentive and CR-Incentive runs) outcome feedback is not necessary to elicit robust striatal response. These findings extend recent work suggesting that perceived agency in the context of feedback is critical for evoking dorsal striatum responses (Tricomi et al., 2004).

Tricomi et al. (2004) demonstrated that caudate activations during an oddball-like paradigm critically depended upon the subjects' perceived agency in the outcomes; prominent caudate activation was observed only when subjects believed (incorrectly) that their responses influenced the subsequent availability of rewards. In the current data, however, perceived agency does not directly account for striatal activation differences, because both recognition memory conclusions, whether "Old" or "New", are under the subject's control. Furthermore, striatal activation was found even in the absence of monetary rewards or confirmation of the availability of reward through feedback. Therefore, the key factor appears to be whether the memory decision corresponds to a preferred outcome. This finding is important because it sug- 
gests that these caudate regions are not coding environmentally given normative reward value, but may instead be coding the subjective value of decisions in light of their relation to goal constructs. The current data strongly suggest that subjects are not neutral with respect to the outcomes of their recognition conclusions during standard procedures, but instead preferentially construe "Old" decisions as goal consistent, hence triggering responses linked to reward processing or anticipation.

This characterization accords well with the recent finding by Clark et al. (2009) of increased striatal response during miss trials in the context of a slot machine task, provided those misses were perceived as "near misses". Thus, there was striatal activation when observers perceived their actions brought them close to achieving a goal, even though no rewards were received and feedback was negative. In the context of the current recognition discrimination task, this goal-dependent framework makes the strong prediction that the HITs $>$ CRs activation pattern could be reversed (in the absence of incentives) by instructing the subjects that the task was not one of "recognizing Old words", but of "detecting New words". Additionally, this predicted framing effect might be further enhanced by linking the framings to socially desirable characteristics. For example, describing the task as "detecting New pictures" and further noting that "accurate detection of novel items is strongly predictive of general intelligence."

The comparison of the striatal responses with those of rostrolateral PFC, a cortical region more traditionally associated with recognition judgments, demonstrated significant functional differences. Left rostrolateral PFC has been linked to successful recognition (McDermott et al., 2000; Donaldson et al., 2001) and contextual memory judgments (Dobbins et al., 2002). There were four characteristics that distinguished the striatal response from that of rostrolateral PFC. First, unlike the striatum, rostrolateral PFC was insensitive to the presence of confirmatory feedback. Second, the region did not demonstrate a full crossover pattern that followed the external incentives during the latter runs. Third, unlike the striatum, rostrolateral PFC was insensitive to individual differences in the reward responsiveness personality trait. Finally, the striatal and rostrolateral regions dissociated in terms of their ability to competitively predict activations in other cortical areas (Table 2, Fig. 5). This pattern of findings implies a clear functional dissociation between the two regions while simultaneously ruling out an interpretation for either region strictly in terms of episodic retrieval success.

Since the current study was not designed to specifically explore rostrolateral PFC our functional claims are more tentative. However, the clear insensitivity of the region to feedback and its unique predictive relationship with dorsolateral PFC and anterior lateral parietal regions during the competitive prediction analysis suggest that it may be important for successful executive control when such control relies upon episodic memory retrieval (Brass et al., 2005; Vincent et al., 2008). Furthermore, the current data suggest that an elevated response in this region may jointly require both the recovery of episodic content and the previously planned use of such content in constraining future behavioral choices. This would explain why the HITs versus CRs response difference was eliminated when incentives highlighted the detection of new items since this would minimize the planning of retrieval-based actions, and this interpretation is consistent with recent findings linking the region to prospective remembering and contingency planning (Haynes et al., 2007; Dumontheil et al., 2008; Reynolds et al., 2009).

In summary, we contrasted retrieval-based and motivational accounts of the differential striatal response to HITs versus CRs observed during standard recognition paradigms. The data favored the latter demonstrating that the striatal activation seen during standard retrieval success contrasts (HITs versus CRs) also tracked extrinsic incentives and in fact qualitatively reversed when "New" conclusions became the a priori favored retrieval outcome. Furthermore, the level activation appeared to be largely independent of the quality of underlying mnemonic evidence since errors also elicited robust activation, provided they were congruent with the incentive conditions (see supplemental data). Additionally, these incentive-contingent responses were seen even in the absence of external feedback, although such feedback amplified the response, and connectivity analyses demonstrated that the striatal responses differed from that in rostrolateral PFC in terms of ability to predict tertiary regions associated with cognitive control (e.g., lateral parietal) and reward processes (e.g., anterior insula). Finally, the striatal response, particularly in ventral striatum, was shown to track individual personality differences in reward reactivity, which provided additional support for a motivational versus retrieval-based interpretation of its role during recognition. These novel findings jointly suggest that the observed striatal activation does not directly depend upon the expectation of extrinsic reinforcement, the receipt of performance feedback, or the successful recovery of episodic information. Instead, the findings are more consistent with a motivational account that contends that the striatal activation marks the convergence of the subjects' goals and their rendered conclusions.

\section{References}

Achim AM, Lepage M (2005) Dorsolateral prefrontal cortex involvement in memory post-retrieval monitoring revealed in both item and associative recognition tests Neuroimage 24:1113-1121.

Adcock RA, Thangavel A, Whitfield-Gabrieli S, Knutson B, Gabrieli JD (2006) Reward-motivated learning: mesolimbic activation precedes memory formation. Neuron 50:507-517.

Belujon P, Grace AA (2008) Critical role of the prefrontal cortex in the regulation of hippocampus-accumbens information flow. J Neurosci 28:9797-9805.

Brass M, Ullsperger M, Knoesche TR, von Cramon DY, Phillips NA (2005) Who comes first? The role of the prefrontal and parietal cortex in cognitive control. J Cogn Control 17:1367-1375.

Brett M, Anton JL, Valabregue R, and Poline JB (2002) Region of interest analysis using an SPM toolbox. Paper presented at the 8th International Conference on Functional Mapping of the Human Brain, Sendai, Japan, June.

Buckner RL, Koutstaal W, Schacter DL, Wagner AD, Rosen BR (1998) Functional-anatomic study of episodic retrieval using fMRI. I. Retrieval effort versus retrieval success. Neuroimage 7:151-162.

Carver CS, White TL (1994) Behavioral inhibition, behavioral activation, and affective responses to impending reward and punishment: the BIS/ BAS Scales. J Pers Soc Psychol 67:319-333.

Christoff K, Gabrieli JDE (2000) The frontopolar cortex and human cognition: evidence for a rostrocaudal hierarchical organization within the human prefrontal cortex. Psychobiology 28:168-186.

Clark L, Lawrence AJ, Astley-Jones F, Gray N (2009) Gambling near-misses enhances motivation to gamble and recruit win-related brain circuitry. Neuron 61:481-490.

Cohen MX, Schoene-Bake JC, Elger CE, Weber B (2009) Connectivitybased segregation of the human striatum predicts personality characteristics. Nat Neurosci 12:32-34.

De Martino B, Kumaran D, Holt B, Dolan RJ (2009) The neurobiology of reference-dependent value computation. J Neurosci 29:3833-3842.

Delgado MR, Locke HM, Stenger VA, Fiez JA (2003) Dorsal striatum responses to reward and punishment: Effects of valence and magnitude manipulations. Cogn Affect Behav Neurosci 3:27-38.

Dobbins IG, Han S (2006) Isolating rule- versus evidence-based prefrontal activity during episodic and lexical discrimination: a functional magnetic resonance imaging investigation of detection theory distinctions. Cereb Cortex 16:1614-1622. 
Dobbins IG, Foley H, Schacter DL, Wagner AD (2002) Executive control during episodic retrieval: multiple prefrontal processes subserve source memory. Neuron 35:989-996.

Donaldson DI, Petersen SE, Buckner RL (2001) Dissociating memory retrieval processes using fMRI: evidence that priming does not support recognition memory. Neuron 31:1047-1059.

Dumontheil I, Burgess PW, Blakemore SJ (2008) Development of rostral prefrontal cortex and cognitive and behavioral disorders. Dev Med Child Neurol 50:168-181.

Elliott R, Friston KJ, Dolan RJ (2000) Dissociable neural responses in human reward systems. J Neurosci 20:6159-6165.

Grahn JA, Parkinson JA, Owen AM (2009) The role of the basal ganglia in learning and memory: neuropsychological studies. Behav Brain Res 199:53-60.

Haynes JD, Sakai K, Rees G, Gilbert S, Frith C, Passingham RE (2007) Reading hidden intentions in the human brain. Curr Biol 17:323-328.

Henson RN, Hornberger M, Rugg MD (2005) Further dissociating the processes involved in recognition memory: an fMRI study. J Cogn Neurosci 17:1058-1073.

Hornberger M, Rugg MD, Henson RN (2006) fMRI correlates of retrieval orientation. Neuropsychologia 44:1425-1436.

Iidaka T, Matsumoto A, Nogawa J, Yamamoto Y, Sadato N (2006) Frontoparietal network involved in successful retrieval from episodic memory. Spatial and temporal analyses using fMRI and ERP. Cereb Cortex 16:1349-1360.

Johnson MK, Hashtroudi S, Lindsay DS (1993) Source monitoring. Psychol Bull 114:3-28.

Knutson B, Fong GW, Adams CM, Varner JL, Hommer D (2001) Dissociation of reward anticipation and outcome with event-related fMRI. Neuroreport 12:3683-3687.

Koechlin E, Summerfield C (2007) An information theoretical approach to prefrontal executive function. Trends Cogn Sci 11:229-235.

Lepage M, Ghaffar O, Nyberg L, and Tulving E (2000) Prefrontal cortex and episodic memory retrieval mode. Proc Natl Acad Sci U S A 97:506-511.

Locke HS, Braver TS (2008) Motivational influences on cognitive control: behavior, brain activation, and individual differences. Cogn Affect Behav Neurosci 8:99-112.

Macmillan NA, Creelman C (1991) Detection theory: a user's guide. New York: Cambridge UP

McDermott KB, Jones TC, Petersen SE, Lageman SK, Roediger HL 3rd (2000) Retrieval success is accompanied by enhanced activation in anterior prefrontal cortex during recognition memory: an event-related fMRI Study. J Cogn Neurosci 12:965-976.

McDermott KB, Szpunar KK, and Christ SE (2009) Laboratory-based and autobiographical retrieval tasks differ substantially in their neural substrates. Neuropsychologia 47:2290-2298.

Nakano K, Kayahara T, Tsutsumi T, Ushiro H (2000) Neural circuits and functional organization of the striatum. J Neurol 247 [Suppl 5]: V1-V5.

O’Doherty J, Dayan P, Schultz J, Deichmann R, Friston K, Dolan RJ (2004) Dissociable roles of ventral and dorsal striatum in instrumental conditioning. Science 304:452-454.

Preuschoff K, Quartz SR, Bossaerts P (2008) Human insula activation reflects risk prediction errors as well as risk. J Neurosci 28:2745-2752.

Reynolds JR, McDermott KB, Braver TS (2006) A direct comparison of an- terior prefrontal cortex involvement in episodic retrieval and integration. Cereb Cortex 16:519-528.

Reynolds JR, West R, Braver T (2009) Distinct neural circuits support transient and sustained processes in prospective memory and working memory. Cereb Cortex 19:1208-1221.

Rissman J, Gazzaley A, D’Esposito M (2004) Measuring functional connectivity during distinct stages of a cognitive task. Neuroimage 23:752-763.

Rugg MD, Wilding EL (2000) Retrieval processing and episodic memory: electrophysiological and neuroimaging evidence. Trends Cogn Sci 4:108-115.

Rugg MD, Fletcher PC, Frith CD, Frackowiak RS, Dolan RJ (1996) Differential activation of the prefrontal cortex in successful and unsuccessful memory retrieval. Brain 119:2073-2083.

Schweinhardt P, Seminowicz DA, Jaeger E, Duncan GH, Bushnell MC (2009) The anatomy of the mesolimbic reward system: a link between personality and the placebo analgesic response. J Neurosci 29:4882-4887.

Shapiro ML, Kennedy PJ, Ferbinteanu J (2006) Representing episodes in the mammalian brain. Curr Opin Neurobiol 16:701-709.

Spaniol J, Davidson PS, Kim AS, Han H, Moscovitch M, Grady CL (2009) Event-related fMRI studies of episodic encoding and retrieval: metaanalyses using activation likelihood estimation. Neuropsychologia 47: 1765-1779.

Tremblay L, Hollerman JR, Schultz W (1998) Modifications of reward expectation-related neuronal activity during learning in primate striatum. J Neurophysiol 80:964-977.

Tricomi E, Fiez JA (2008) Feedback signals in the caudate reflect goal achievement on a declarative memory task. Neuroimage 41:1154-1167.

Tricomi EM, Delgado MR, Fiez JA (2004) Modulation of caudate activity by action contingency. Neuron 41:281-292.

Tulving E, Kapur S, Markowitsch HJ, Craik FI, Habib R, Houle S (1994) Neuroanatomical correlates of retrieval in episodic memory: auditory sentence recognition. Proc Natl Acad Sci U S A 91:2012-2015.

van der Meer MA, Redish AD (2009) Covert expectation-of-reward in rat ventral striatum at decision points. Front Integr Neurosci 3:1-15.

Van Essen DC (2005) A population-average, landmark- and surface-based (PALS) atlas of human cerebral cortex. Neuroimage 28:635-662.

Vincent JL, Kahn I, Snyder AZ, Raichle ME, Buckner RL (2008) Evidence for a frontoparietal control system revealed by intrinsic functional connectivity. J Neurophysiol 100:3328-3342.

von Zerssen GC, Mecklinger A, Opitz B, von Cramon DY (2001) Conscious recollection and illusory recognition: an event-related fMRI study. Eur J Neurosci 13:2148-2156.

Wager TD, Nichols TE (2003) Optimization of experimental design in fMRI: a general framework using a genetic algorithm. Neuroimage 18:293-309.

Wendelken C, Nakhabenko D, Donohue SE, Carter CS, Bunge SA (2008) "Brain is to thought as stomach is to ??": Investigating the role of rostrolateral prefrontal cortex in relational reasoning. J Cogn Neurosci 20:682-693.

Wittmann BC, Schott BH, Guderian S, Frey JU, Heinze HJ, and Düzel E (2005) Reward-related fMRI activation of dopaminergic midbrain is associated with enhanced hippocampus-dependent long-term memory formation. Neuron 45:459-467.

Zink CF, Pagnoni G, Martin ME, Dhamala M, Berns GS (2003) Human striatal response to salient nonrewarding stimuli. J Neurosci 23:80928097. 